\title{
Diacronie
}

Studi di Storia Contemporanea

$N^{\circ} 33,1 \mid 2018$

Guerra e pace

\section{Matteo Loconsole, Storia della contraccezione in Italia tra falsi moralisti, scienziati e sessisti}

\section{Sandro Bellassai}

\section{Q OpenEdition \\ 1 Journals}

Edizione digitale

URL: http://journals.openedition.org/diacronie/7509

DOI: $10.4000 /$ diacronie.7509

ISSN: 2038-0925

Editore

Association culturelle Diacronie

Notizia bibliografica digitale

Sandro Bellassai, « Matteo Loconsole, Storia della contraccezione in Italia tra falsi moralisti, scienziati e sessisti », Diacronie [Online], № 33, 1 | 2018, documento 10, Messo online il 29 mars 2018, consultato il 24 septembre 2020. URL : http://journals.openedition.org/diacronie/7509 ; DOI : https://doi.org/ $10.4000 /$ diacronie. 7509 


\section{Diacronie}

Studi di Storia Contemporanea

\section{$33,1 / 2018$}

Guerra e pace: declinazioni politiche, sociali e culturali del conflitto in età contemporanea

\section{RECENSIONE: Matteo LOCONSOLE, Storia della contraccezione in Italia tra falsi moralisti, scienziati e sessisti, Bologna, Pendragon, 2017, 153 pp.}

Per citare questo articolo:

BELLASSAI, Sandro, «RECENSIONE: Matteo LOCONSOLE, Storia della contraccezione in Italia tra falsi moralisti, scienziati e sessisti, Bologna, Pendragon, 2017, 153 pp.», Diacronie. Studi di Storia Contemporanea : Guerra e pace: declinazioni politiche, sociali e culturali del conflitto in età contemporanea, 33, 1/2018, 29/03/2018,

URL: < http://www.studistorici.com/2018/03/29/bellassai_numero_33/ >

Diacronie Studi di Storia Contemporanea $\rightarrow$ http://www.diacronie.it

Rivista storica online. Uscita trimestrale.

redazione.diacronie@hotmail.it

Comitato di direzione: Naor Ben-Yehoyada - João Fábio Bertonha - Christopher Denis-Delacour - Maximiliano Fuentes Codera Anders Granås Kjøstvedt - John Paul Newman - Deborah Paci - Niccolò Pianciola - Spyridon Ploumidis - Wilko Graf Von Hardenberg

Comitato di redazione: Jacopo Bassi - Luca Bufarale - Gianluca Canè - Luca G. Manenti - Fausto Pietrancosta - Alessandro Salvador - Matteo Tomasoni - Luca Zuccolo 


\title{
10/ RECENSIONE: Matteo LOCONSOLE, Storia della contraccezione in Italia tra falsi moralisti, scienziati e sessisti, Bologna, Pendragon, 2017, 153 pp.
}

\author{
A cura di Sandro BELLASSAI
}

Nell'Italia di inizio Novecento, come altrove, il dibattito sulla questione del controllo delle nascite si inquadrò all'interno di una pluridecennale vocazione della scienza e della cultura occidentali alla razionale "gestione del patrimonio biologico della collettività» ${ }^{1}$. In tale orizzonte biopolitico, che prese forma a partire almeno dalla metà dell'Ottocento, confluirono teorie, linguaggi, posizioni ideologiche e istanze normative molto eterogenee, e si intrecciarono dinamiche di mutamento politiche, sociali e istituzionali diverse ma ugualmente importanti. L'idea della possibilità di una "selezione artificiale» delle future generazioni umane, in particolare, nasceva sulla scia delle ipotesi darwiniane in merito alla «selezione naturale» delle specie; la sua legittimazione politica si fondava sul duplice presupposto di una necessaria "correzione" del cammino biologico dell'umanità alla luce delle sue preoccupanti tendenze alla «degenerazione», da un lato, e di una nuova funzione ieratica della scienza in generale e della classe medica in special modo, dall'altro.

Il crescente successo politico dei concetti di Razza e Nazione nella Belle Époque, d'altro canto, spinse molto spesso alla rappresentazione drammatica di una collettività "organica" minacciata da gravi pericoli interni ed esterni, di fronte ai quali si giustificavano non solo un perentorio interventismo pedagogico in nome della Legge e della Verità (incarnata non metafisicamente dal verbo positivista), ma anche un'opera complessiva di disciplinamento, inquadramento autoritario, vero e proprio rimodellamento drastico - al limite - dei corpi e delle anime degli esseri umani moderni. Era questo un paradigma delle relazioni sociali ampiamente trasversale sul piano ideologico: anche coloro i quali non si iscrivevano affatto al fronte dell'ordine e dell'autorità, come socialisti, democratici, progressisti vari, vagheggiavano comunque, non di

\footnotetext{
${ }^{1}$ MANTOVANI, Claudia, Rigenerare la società. L'eugenetica in Italia dalle origine ottocentesche agli anni Trenta, Soveria Mannelli, Rubbettino, 2004, p. 89.
} 
rado, un intrusivo progetto «rigeneratore» di una società disordinata e sofferente quanto ai suoi assetti sanitari, etici, familiari, in nome di una prevalente ragione dell'interesse collettivo su qualsivoglia diritto individuale.

Gli apostoli delle nuove discipline dell'eugenetica, dell'igiene, della medicina sociale guardavano all'insieme dei comportamenti privati, fin nella più intima sfera sessuale, come a fenomeni di grande rilevanza sociale, e quindi più che legittimamente suscettibili di interventi normativi e "correttivi" da parte delle agenzie pubbliche e della scienza in generale. Questa “politicizzazione" delle questioni riproduttive sottintendeva un'idea del «corpo» sociale come organismo da sottoporre a una vera e propria ortopedia autoritaria, ma non va comunque confusa teleologicamente con gli sviluppi più tragici della storia novecentesca; per quanto, è inutile dirlo, diverse familiarità culturali è possibile rintracciare fra un certo darwinismo tardo-ottocentesco che teorizzava di lasciare gli "inetti" al loro destino infausto, le leggi statunitensi di sterilizzazione (a partire dal 1906) degli esseri umani più imperfetti, e l'abominio dell'Aktion T-4 operante nel Terzo Reich.

Di tutto questo non può certamente essere accusato il pastore inglese Thomas Malthus, fondatore della demografia moderna, che nel 1798 aveva posto energicamente il problema sociale del rapporto fra popolazione e risorse; né possono esserlo i cosiddetti neomalthusiani, che dalla seconda metà dell'Ottocento, in vari paesi occidentali, propagandarono la causa della "generazione cosciente», e dunque caldeggiarono la diffusione di metodi contraccettivi di varia natura. Più che a una Storia della contraccezione in Italia, come suggerirebbe il suo titolo, è a questo scenario specifico che l'opera di Loconsole è dedicata. Nel primo capitolo del volume, l'autore ${ }^{2}$ tratta delle questioni aperte dalla diffusione nella cultura europea ottocentesca delle teorie di Malthus e di Darwin, dell'eugenetica, di una vera e propria «utopia igienica»: tendenze, queste, che nel loro insieme ponevano tutte al centro i problemi della riproduzione umana e del rafforzamento biologico della popolazione. Il secondo capitolo è in buona parte dedicato a delineare il movimento neomalthusiano italiano, nel primo quindicennio del Novecento, e le reazioni aspramente polemiche che esso si attirò dal campo perbenista. Infine, nell'ultimo capitolo si ricostruiscono alcune vicende e contenuti della rivista neomalthusiana «L'educazione sessuale», certamente un caso interessante e poco conosciuto di divulgazione scientifica laica e progressista in materia di sessualità.

Il libro di Loconsole si pone quindi l'obiettivo di dar conto del movimento neomalthusiano nell'Italia di inizio Novecento, evidenziandone opportunamente le radici culturali nella scienza europea ottocentesca nonché documentando con ampiezza la fierissima avversione nei suoi confronti da parte, principalmente, di esponenti autorevoli del moralismo cattolico. Costoro

\footnotetext{
2 Matteo Loconsole, laureato in filosofia all'Università di Bologna, è attualmente dottorando presso l'Università di Roma Tre.
} 
tuonarono costantemente contro la «frode della generazione», com'era facile attendersi, trovando anche ampi consensi nella magistratura (che nel 1912 e nel 1913 precettò in tribunale gli autori dei due testi neomalthusiani più importanti in Italia, quelli di Secondo Giorni e di Ettorina Cecchi, con l'accusa di oltraggio al pudore, ma infine assolvendoli). Promosso da vari democratici, socialisti e anarchici, il neomalthusianesimo incontrò allo stesso tempo una decisa opposizione anche a sinistra, non solo in Italia: contrari o diffidenti si dichiararono infatti negli anni personaggi illustri come Kropotkin, Lenin, Sorel, Salvemini, Prezzolini, Mondolfo e vari altri.

Oltre a perseguire la missione tardo-illuministica di sottrarre a medievali tenebre $\mathrm{i}$ fatti sessuali, di cui non si stancavano di sottolineare le moderne valenze sociali, i neomalthusiani si prefiggevano l'obiettivo di migliorare - grazie alla possibilità di scegliere razionalmente se e quanti figli avere - le condizioni materiali e morali delle famiglie proletarie, e anche, nello specifico, delle donne del popolo. Se per la dottoressa Cecchi, infatti, la «maternità libera» rappresentava una condizione imprescindibile dell'emancipazione femminile ${ }^{3}$, per un altro medico neomalthusiano, Luigi Berta, la limitazione del numero delle nascite era necessaria allo scopo di «sollevare il tenore di vita delle classi operaie» ${ }^{4}$. Ma il movimento per la «procreazione responsabile», in accordo con un più generale orientamento eugenetico (i cui esponenti, tuttavia, non sempre ne approvavano metodi e pratiche), si proponeva anche un miglioramento qualitativo della specie umana: «I poveri sono fecondi, ma la loro fecondità è malsana e inutile, perché la miseria diventa per queste classi prolifiche il substrato di malattie essenzialmente popolari: la tubercolosi, la sifilide, l'alcoolismo», argomentava infatti lo stesso Berta ${ }^{5}$.

$\mathrm{Al}$ di là e ben prima della effimera popolarità della propaganda neomalthusiana (inquadrabile cronologicamente all'indomani del convegno vociano sulla "questione sessuale" del 1910), tuttavia, la limitazione delle nascite doveva essere in Italia una pratica già abbastanza diffusa, se è vero che il tasso di natalità era sceso nel 1914 al 31 per mille, dal 38 di trent'anni prima ${ }^{6}$. Molto presto, d'altronde, nel montante clima nazionalista che preannunciava la catastrofe bellica, e sotto l'influenza del dibattito francese sul calo demografico come indebolimento della potenza nazionale, la causa del controllo delle nascite venne sempre più ampiamente stigmatizzata come politicamente deleteria. Fu soprattutto per tale ragione che nel 1915, a solo un anno e mezzo dal suo esordio, sospese le pubblicazioni la testata «L'educazione sessuale. Rivista di neomalthusianismo e di eugenica», la cui nascita era stata simultanea al tardivo coronamento organizzativo del movimento, con la fondazione della Lega italiana neomalthusiana (in Gran

\footnotetext{
${ }^{3}$ LOCONSOLE, Matteo, Storia della contraccezione in Italia tra falsi moralisti, scienziati e sessisti, Bologna, Pendragon, 2017, p. 66.

${ }^{4}$ Ibidem, p. 50.

${ }^{5}$ Cit. ibidem, p. 51.

${ }^{6}$ RIFELLI, Giorgio, Sessualità: nascita di un concetto e di una disciplina, in RIFELLI, Giorgio, ZIGLIO, Corrado, Per una storia dell'educazione sessuale 1870-1920, Firenze, La Nuova Italia, 1991, pp. 9-105, p. 78.
} 
Bretagna un'organizzazione analoga era stata creata già nel 1878, in Francia nel 1896). La valorizzazione analitica di questa fonte è uno dei tratti originali della ricerca di Loconsole, che si diffonde efficacemente anche sui rapporti fra la rivista stessa e scienziati di orientamento eugenetico, femministe, studiosi ed educatori progressisti. Poco approfondite, tuttavia, risultano in quest'opera le relazioni fra il neomalthusianesimo italiano e quello internazionale, al punto ma è solo un esempio - che non vi si trova nemmeno notizia dell'affiliazione della Lega italiana alla Federazione universale della rigenerazione umana, con sede a Parigi. Neppure un cenno l'autore dedica inoltre a coeve esperienze italiane, che pure furono piuttosto attive nel diffondere orientamenti analoghi: basti pensare a un'altra rivista fondata nel 1914, milanese e diretta dal medico Felice Marta, ugualmente intitolata «L'educazione sessuale», o alle attività dell'Istituto "Il Pensiero", fiorentino, che pubblicava opere della stessa Ettorina Cecchi e addirittura commercializzava direttamente prodotti contraccettivi ${ }^{7}$.

Mentre si dilunga sulle posizioni degli avversari cattolici del movimento, come Rodolfo Bettazzi (infaticabile censore della propaganda neomalthusiana, che il presidente della Lega per la pubblica moralità condannava come attività pornografica, disgregatrice e sommamente immorale), anche a causa della sua brevità l'opera di Loconsole tratta invece sbrigativamente ma talvolta anche discutibilmente - una serie di importanti questioni interpretative legate al contesto culturale e politico dell'epoca. $\mathrm{E}$ da questo punto di vista è purtroppo significativo che autori come George Mosse, che sul rapporto fra nazionalismo e sfera sessuale ha pionieristicamente aperto prospettive critiche ancora oggi imprescindibili ${ }^{8}$, qui non siano stati neppure presi in considerazione. Ma non è questa l'unica carenza, sempre rimanendo sul piano interpretativo, di una ricerca che, pur dotata di alcuni meriti, forse si sarebbe giovata di una riflessione ulteriormente meditata ed estesa. La necessità analitica di distinguere fra positivismo e positivismo «applicato», per citare un altro esempio, laddove solo al secondo si imputano pregiudizi e vizi ideologici, appare quantomeno dubbia. Così come poco chiara appare l'urgenza di separare "virtuisti" e "studiosi", i primi volendo a tutti i costi trovare nella scienza conferma a idee preconcette, $\mathrm{i}$ secondi invece che, «pur rimanendo inevitabilmente, e forse inconsapevolmente, coinvolti dall'ormai vigente morale sessuale, cercarono di emanciparsi da essa, servendosi della scienza come di uno strumento per debellare antichi pregiudizi senza alcun fondamento reale» ${ }^{9}$. Mai come nell'età del positivismo, forse, presunzione di oggettività e pregiudizio (di classe, di genere, di "razza") apparvero come due facce della stessa medaglia:

\footnotetext{
${ }^{7}$ WANROOIJ, Bruno P. F., Storia del pudore. La questione sessuale in Italia 1860-1940, Venezia, Marsilio, 1990, pp. 77-78.

${ }^{8}$ Cfr. MOSSE, George L., Sessualità e nazionalismo. Mentalità borghese e rispettabilità, Roma-Bari, Laterza, 1996 [Ed. originale: Nationalism and sexuality. Respectability and abnormal sexuality in modern Europe, New York, Howard Fertig, 1985].

${ }^{9}$ LOCONSOLE, Matteo, op. cit., pp. 107-108.
} 
quella stessa medaglia che le borghesie occidentali si appuntarono da sé stesse, trionfalmente, sul proprio petto virile. 


\section{L'AUTORE}

Sandro BELLASSAI insegna Storia contemporanea e Storia di genere presso l'Università di Bologna. Ha condotto ricerche sulle relazioni di genere e sulle culture politiche in età contemporanea. Ha curato, con Maria Malatesta, Genere e mascolinità. Uno sguardo storico (Roma, Bulzoni, 2000). È autore, fra l'altro, di: La morale comunista. Pubblico e privato nella rappresentazione del Pci (1947-1956) (Roma, Carocci, 2000, vincitore del Premio Sissco 2001); L'invenzione della virilità. Politica e immaginario maschile nell'Italia contemporanea (Roma, Carocci, 2011).

URL: < http://www.studistorici.com/progett/autori/\#Bellassai > 